\title{
Estudio anatómico e histológico de las arterias que irrigan el páncreas e intestino delgado de la alpaca (Vicugna pacos)
}

\author{
Anatomical and histological study of the arteries that irrigate the pancreas and \\ small intestine of the alpaca (Vicugna pacos)
}

Rose Barreto R..$^{1,3}$, Alberto Sato S., ${ }^{1,4}$, Marcelo Daniel Ghezzi ${ }^{2}$, Francisco Santos R. ${ }^{1}$, Alexander Chávez R. ${ }^{1}$, Miluska Navarrete Z. ${ }^{1}$

\section{Resumen}

Este estudio describe las arterias que irrigan el páncreas e intestino delgado de la alpaca mediante disección, angiografía, repleción vascular e histología. Se emplearon seis alpacas, de las cuales cuatro fueron mayores de dos años y dos de cuatro meses. Macroscópicamente, el páncreas estuvo irrigado principalmente por las arterias pancreática duodenal craneal y caudal; además, ramas provenientes de la arteria hepática, arteria esplénica y la rama de la primera arteria yeyunal. Estas arterias y ramas ingresaron al páncreas (porción izquierda, derecha y cuerpo) formando anastomosis en forma de redes, en el interior del parénquima. Las técnicas histológicas evidenciaron que las capas de las arterias de la alpaca presentan dos capas, compacta y esponjosa, conformando la túnica adventicia de las arterias pancreática duodenal craneal y caudal, celíaca y mesentérica craneal. La arteria pancreática duodenal caudal tuvo la capa adventicia compacta más gruesa, siendo esta arteria la que irriga el lóbulo derecho pancreático, parte del lóbulo izquierdo y el duodeno.

Palabras clave: alpaca; anatomía; arteria; histología; páncreas; yeyuno

${ }^{1}$ Laboratorio de Anatomía Animal y Fauna Silvestre, Facultad de Medicina Veterinaria, Universidad Nacional Mayor de San Marcos, Lima, Perú

${ }^{2}$ Laboratorio de Anatomía, Facultad de Ciencias Veterinarias, Universidad Nacional del Centro de la Provincia de Buenos Aires; Tandil, Argentina

${ }^{3}$ E-mail: belisama_vet@yahoo.es

${ }^{4}$ E-mail: albertosato2000@yahoo.com

Recibido: 12 de junio de 2018

Aceptado para publicación: 24 de noviembre de 2018 


\section{Abstract}

This study describes the arteries that irrigate the pancreas and small intestine of the alpaca by dissection, angiography, vascular repletion and histology. Six alpacas were used, of which four were older than two years and two were four months old. Macroscopically, the pancreas was mainly irrigated by the pancreatic duodenal cranial and caudal arteries; in addition, branches from the hepatic artery, splenic artery and the branch of the first jejunal artery. These arteries and branches entered the pancreas (left, right and body portions) forming anastomosis in the form of nets, inside the parenchyma. Histological techniques showed that alpaca arteries have two layers, compact and spongy, forming the adventitia tunic of the duodenal cranial and caudal pancreatic arteries, celiac and cranial mesenteric arteries. The caudal duodenal pancreatic artery had the thickest compact adventitial layer, this artery supplying the right pancreatic lobe, part of the left lobe and the duodenum.

Key words: alpaca; anatomy; artery; histology; jejunum; pancreas

\section{INTRODUCCIÓN}

La alpaca (Vicugna pacos) es un camélido de gran importancia en los países altoandinos. A pesar de vivir en medios hostiles, su adaptación ha sido exitosa, ya que puede soportar bajos niveles de oxígeno y aprovechar la vegetación y medio ambiente para desarrollarse. Hoy en día el estudio de los diferentes sistemas que forman el organismo de la alpaca está en aumento debido a los beneficios económicos que otorga en fibra y carne, así como fisiológicamente, por su respuesta lenta y moderada a la insulina, de forma similar a la diabetes humana (Cebra et al., 2001), pero sin verse afectada.

Se han realizado varios estudios descriptivos de algunos órganos de la alpaca, tales como el sistema muscular, músculos del miembro torácico, músculos del cuello (Mendoza y Montoya, 2002), circunvoluciones y giros cerebrales (Sato, 1970), cerebelo (Sato y Kian, 1976), plexo braquial (Sato et al., 1983), irrigación del abdomen, irrigación del estómago (Laos, 1973), irrigación del intesti- no delgado (Olivos, 1974) e irrigación de los órganos mesentéricos (Santos et al., 2015).

Respecto a la irrigación del páncreas, Lis et al. (2003) mencionan que el aporte sanguíneo de oxígeno al páncreas es variado e importante, pues recibe ramas de la arteria celíaca de modo directo y a través de sus ramas gástrica izquierda, hepática y esplénica. Además, existe una arteria pancreáticoduodenal. Al mismo tiempo, recibe sangre arterial aportada por la arteria mesentérica craneal por medio de una rama llamada pancreático-duodenal caudal. Una particularidad del modelo circulatorio del páncreas es que la capilarización arterial recorre primero los islotes y luego se deriva y baña los acinos exocrinos, de tal manera que las células secretoras de jugo pancreático reciben la sangre con más alto tenor hormonal de insulina y glucagón. Se ha comprobado que este hecho influye en el trofismo y función exocrina de modo estimulante (Lis et al., 2003). Por lo tanto, el objetivo del presente trabajo fue describir macroscópica y microscópicamente la angiología arterial del páncreas e intestino delgado de alpacas. 


\section{Materiales y Métodos}

\section{Animales y Muestras}

Fueron utilizadas seis alpacas (Vicugna pacos), cuatro adultos mayores de 2 años y dos crías de 4 meses, de raza Huacaya, obtenidas de la Estación Maranganí del Centro de Investigación IVITA, ubicada en Cusco, Perú. El estudio se realizó en el Laboratorio de Anatomía Animal y Fauna Silvestre de la Facultad de Medicina Veterinaria (FMV), Universidad Nacional Mayor de San Marcos (UNMSM), Lima. Todos los protocolos de trabajo con los animales fueron aprobados por el Comité de Ética de la FMV-UNMSM (Constancia de Autorización Ética 2017-002).

Las alpacas fueron sedadas utilizando acepromazina maleato $2 \mathrm{mg} / \mathrm{kg}$, vía la vena yugular. Como anestésico se utilizó pentobarbital sódico $10 \mathrm{mg} / \mathrm{kg}$, vía endovenosa, según el protocolo de Cooney et al. (2012). Inmediatamente después de la muerte se realizó una incisión en la superficie ventrolateral del tercio medio del cuello para exponer la vena yugular y arteria carótida para realizar el desangrado. El tracto digestivo, hígado y arteria aorta abdominal de una alpaca adulta y dos crías, fueron congeladas para su posterior procesamiento.

Dos alpacas adultas fueron conservadas mediante formolización, por lo que se inyectó una solución preparada $(200 \mathrm{ml}$ de formol 40\%,100 ml de ácido fénico líquido a $37^{\circ} \mathrm{C}$, glicerina $100 \mathrm{ml}, 600 \mathrm{ml}$ de alcohol $70 \%$ ), a través de una cánula metálica insertada en la arteria carótida, empleando una bomba de vacío regulada con un manómetro de mercurio. Después de 10 días se hizo la disección. Se seccionaron muestras de arterias de $1 \mathrm{~cm}$ de longitud a nivel del origen arterial e ingreso de la arteria al páncreas y yeyuno. Estas muestras se mantuvieron en formol tamponado al 10\%.

La cuarta alpaca adulta fue empleada para realizar la técnica de repleción vascular.
A través de un extremo de la arteria carótida se inyectaron $100 \mathrm{ml}$ de agua destilada para dilatar la arteria y arrastrar restos de sangre. Posteriormente se aplicaron $120 \mathrm{ml}$ de pintura roja de látex, dejando reposar por siete días antes de su disección.

\section{Angiografía}

En una de las alpacas conservadas en formol se realizó una laparotomía por la línea media desde el cartílago xifoides hasta el inicio de la pelvis, hasta ubicar la arteria aorta abdominal, en la cual se insertó una cánula y se inyectaron $90 \mathrm{ml}$ de solución de contraste radiológico yodado al $30 \%$. Así mismo, en las vísceras congeladas de una de las crías se realizó el procedimiento de formolización posterior a su descongelación y en la arteria aorta abdominal se inyectaron $60 \mathrm{ml}$ de solución de contraste radiológico yodado al $30 \%$. Luego se realizaron radiografías y se leyeron empleando un negatoscopio.

\section{Repleción Vascular}

En la otra alpaca adulta conservada en formol se diseccionó la arteria carótida a nivel del cuello, insertando una cánula en la que se inyectaron $120 \mathrm{ml}$ de agua destilada a $37^{\circ} \mathrm{C}$ para lavar y dilatar los vasos sanguíneos. Posteriormente se inyectaron $120 \mathrm{ml}$ de pintura roja de látex para el teñido de las arterias. Se dejó reposar 10 días para favorecer el endurecimiento de la pintura. Luego de ese lapso se realizó una laparotomía por la línea media, se elevó la piel y músculos de la pared abdominal descubriendo las vísceras abdominales. Se diseccionaron las arterias que irrigan el páncreas y yeyuno para su descripción macroscópica empleando la Nómina Anatómica Veterinaria (ICVGAN, 2012).

\section{Histología}

Se tomaron muestras arteriales frescas de $1 \mathrm{~cm}$ de las arterias pancreático duodenal craneal, pancreático duodenal caudal y yeyunales a nivel del origen de la arteria y de su ingreso al páncreas y yeyuno. Las mues- 
tras fueron conservadas en formol tamponado al 10\% hasta su procesamiento (Cediel et al., 2009). Luego de la fijación en parafina, se realizaron cortes de $5 \mu \mathrm{m}$ de espesor y se tiñeron con hematoxilina-eosina. También se utilizó la tinción de tricrómico de Masson para diferenciar las capas arteriales. La lectura de las láminas se realizó con un microscopio binocular Leica DM750 con cámara digital ICC50 HD incorporada y software de medición LAS 4.7.

\section{Resultados y Discusión}

Macroscópicamente, se observó el páncreas de alpaca de color rosado claro, compuesto por lóbulo izquierdo, lóbulo derecho y cuerpo. El cuerpo formaba una «Y» invertida. El lóbulo izquierdo estaba más desarrollado. La irrigación arterial del páncreas de alpaca se realiza principalmente por las arterias pancreática duodenal craneal y pancreática duodenal caudal. También se observó aporte arterial de la primera rama de la arteria yeyunal hacia el lóbulo derecho, así como pequeñas ramas cortas provenientes de la arteria gastroduodenal en su recorrido sobre el cuerpo del páncreas y de la arteria esplénica hacia el lóbulo izquierdo del páncreas (Figuras 1 y 2). Un hallazgo similar se realizó por Navarrete et al. (2015). También, un hallazgo interesante es el origen de la arteria mesentérica craneal, pues en tres de los animales emergió de un mismo tronco y en los otros tres animales emergió de forma separada, lo cual coincide con lo descrito por Olivos (1974).

De la arteria aorta abdominal emergió la arteria celíaca. De esta emergieron tres ramas: la arteria hepática, de la cual la rama gastroduodenal dio origen a la arteria gastroepiploica derecha y a la arteria pancreática duodenal craneal, la cual discurrió hacia caudal emitiendo ramitas cortas hacia el borde anti-mesentérico del duodeno, introduciéndose en su capa muscular y hacia el lóbulo derecho del páncreas, donde se introdujo en su parénquima uniéndose internamente con otras ramas formando redes arteriales. La arteria mesentérica craneal emergió a continuación de la arteria celíaca. Se halló una variación a este nivel ya que en tres animales emergieron de un mismo tronco y en tres de forma separada (Figura 3).

De la arteria celíaca emergieron la arteria pancreática duodenal caudal y la arteria yeyunal. La arteria pancreática duodenal caudal se introduce entre el parénquima del lóbulo derecho y emerge dirigida hacia craneal emitiendo ramas pequeñas cortas en su camino, hasta unirse con la arteria pancreática duodenal craneal, ingresando a nivel de la flexura duodenal caudal al lóbulo derecho del páncreas, donde se anastomosa en el tercio caudal con la arteria pancreático duodenal craneal.

De la primera arteria yeyunal se observó una rama muy delgada que se dirigió hacia el lóbulo derecho del páncreas introduciéndose en su parénquima. La irrigación del yeyuno se inició con la salida de la arteria yeyunal desde la arteria mesentérica craneal, siendo vecina de la arteria pancreática duodenal caudal. La arteria yeyunal se dirigió hacia el borde mesentérico del yeyuno, formando arcos y emitiendo ramas cada vez más pequeñas que se introdujeron en el borde mesentérico hacia su interior (Figura 4).

La angiografía del abdomen de la alpaca logró visualizar las arterias que irrigan el páncreas y el yeyuno (Figura 5).

El origen de las arterias pancreática duodenal caudal y la arteria yeyunal es similar a otros estudios realizados en alpacas (Panesi, 2016) y otros mamíferos domésticos (Evans y De Lahunta, 2002; Dyce et al., 2007). Por lo tanto, la obliteración de alguna rama arterial pequeña del páncreas no ocasionaría alteraciones significativas debido a la presencia de múltiples ramas que se anastomosan entre sí, aunque no se puede aseverar qué porcentaje del páncreas es 


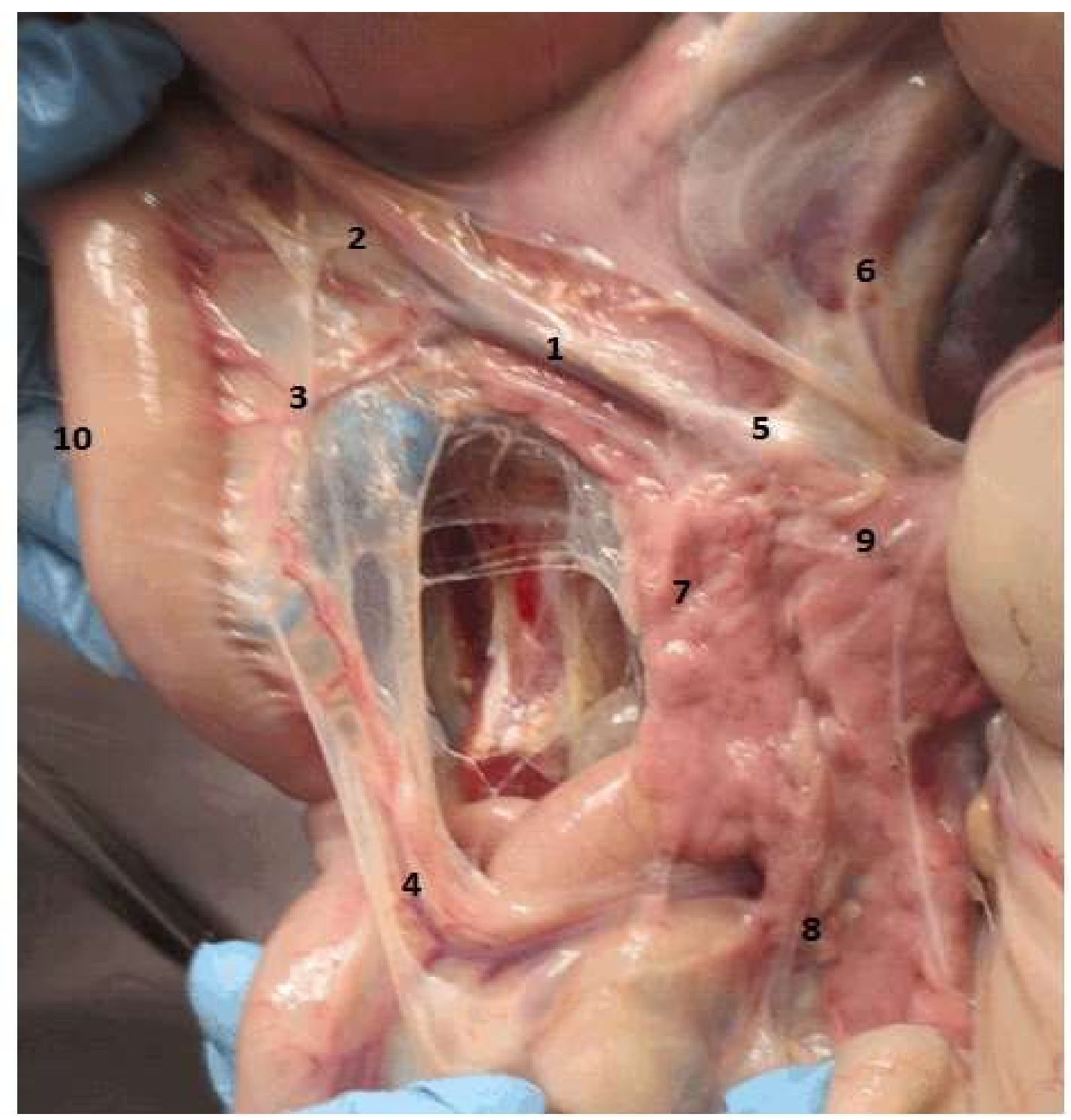

Figura 1. El páncreas de la alpaca y las arterias que lo irrigan. (1) Arteria hepática y arteria gastroduodenal; (2) arteria gastroepiploica derecha; (3) arteria pancreático duodenal craneal; (4) arteria pancreático duodenal caudal; (5) arteria tronco celiaco; (6) arteria aorta abdominal; (7) cuerpo del páncreas; (8) lóbulo derecho del páncreas; (9) lóbulo izquierdo del páncreas; (10) duodeno

irrigado por cada rama arterial debido a la formación de redes arteriales a través de todo el parénquima pancreático.

Histológicamente, las arterias que irrigan al páncreas corresponden a arterias musculares o de mediano calibre, como la arteria pancreática duodenal craneal (Figura 6). Las arterias de mediano calibre son vasos de distribución que permiten la distribución selectiva de sangre a distintos órganos en respuesta a la demanda funcional (Gázquez y Rodríguez, 2004; Kierszenbaum y Tres, 2013). Las muestras arteriales tomadas del 


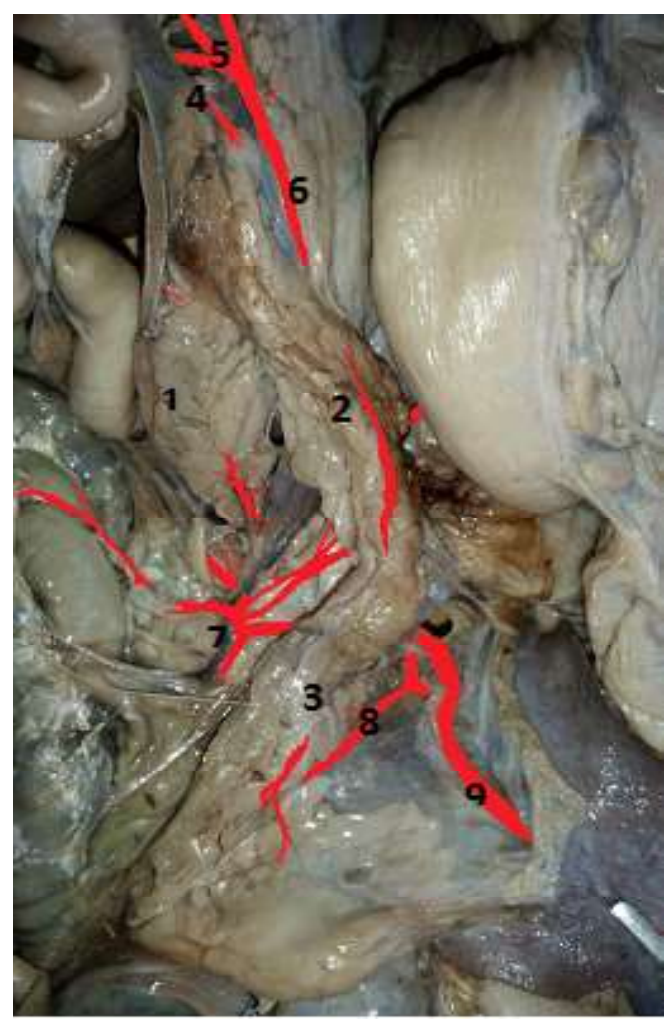

Figura 2. Principales arterias que irrigan al páncreas de la alpaca. (1) Lóbulo derecho; (2) cuerpo del páncreas; (3) lóbulo izquierdo; (4) a. pancreática duodenal craneal; (5) a. gastro-epiploica derecha; (6) a. gastroduodenal; (7) a. yeyunal; (8) a. pancreática duodenal caudal; (9) a. esplénica. Muestra sometida a la técnica de repleción vascular

origen de la arteria e ingreso al páncreas, sometidos a la tinción de Hematoxilina Eosina evidenciaron sobre la superficie arterial una monocapa constituida por células de forma alargadas y núcleo central, dispuestas una a continuación de otra, las cuales conformaron una cubierta endotelial.

A continuación, se apreció la capa media de la arteria (muscular) de mayor grosor, constituida por tejido muscular liso, caracterizado por la presencia de núcleos alargados, de afinidad tintorial basófilos y presencia de capilares sanguíneos. A nivel de la capa ad- venticia de las arterias pancreática duodenal craneal y caudal, arteria mesentérica craneal y tronco celiaco se observó que estaba constituida a su vez por dos capas, una capa de tejido denso rosáceo similar a una capa muscular y otra capa más externa de tejido laxo (Figura 7).

Para diferenciar las capas arteriales se empleó la tinción de tricrómico de Masson, observándose claramente la capa muscular media y la presencia de dos capas que conforman la túnica adventicia. Una más densa y compacta, constituida por tejido conjuntivo con presencia de abundantes fibras colágenas coloreadas de azul y fibras elásticas rojizas, así como vasa vasorum, en el cual los núcleos se observaron de color lila (Figura 8) y una capa laxa de tejido conjuntivo esponjoso, como se aprecia en las figuras 9 y 10 . La arteria pancreática duodenal caudal presentó la capa más compacta y de mayor espesor, con presencia de una segunda capa esponjosa en la túnica adventicia. También se halló una doble capa adventicia a nivel de la arteria mesentérica craneal y tronco celíaco, observándose, además, una capa densa y una laxa. La arteria yeyunal y la rama yeyunal que irrigan el páncreas no presentaron la segunda capa de túnica adventicia. Los hallazgos fueron similares en todas las alpacas.

Las principales arterias pancreática duodenal craneal, pancreática duodenal caudal y mesentérica craneal que irrigan el páncreas de la alpaca, al presentar dos capas diferenciadas en su túnica adventicia, tendrían un papel importante en las funciones nutricionales y de control arterial a bajos niveles de oxígeno, según lo indicado por Cabrera (2008). De esta manera realizan en forma eficiente el aporte de sangre oxigenada al páncreas, cuya capilarización arterial recorre primero los islotes y luego se deriva y baña los acinos exocrinos. Por lo tanto, las células secretoras de jugo pancreático son las que reciben la sangre con más alto tenor hormonal de insulina y glucagón (Lis et al., 2003). 


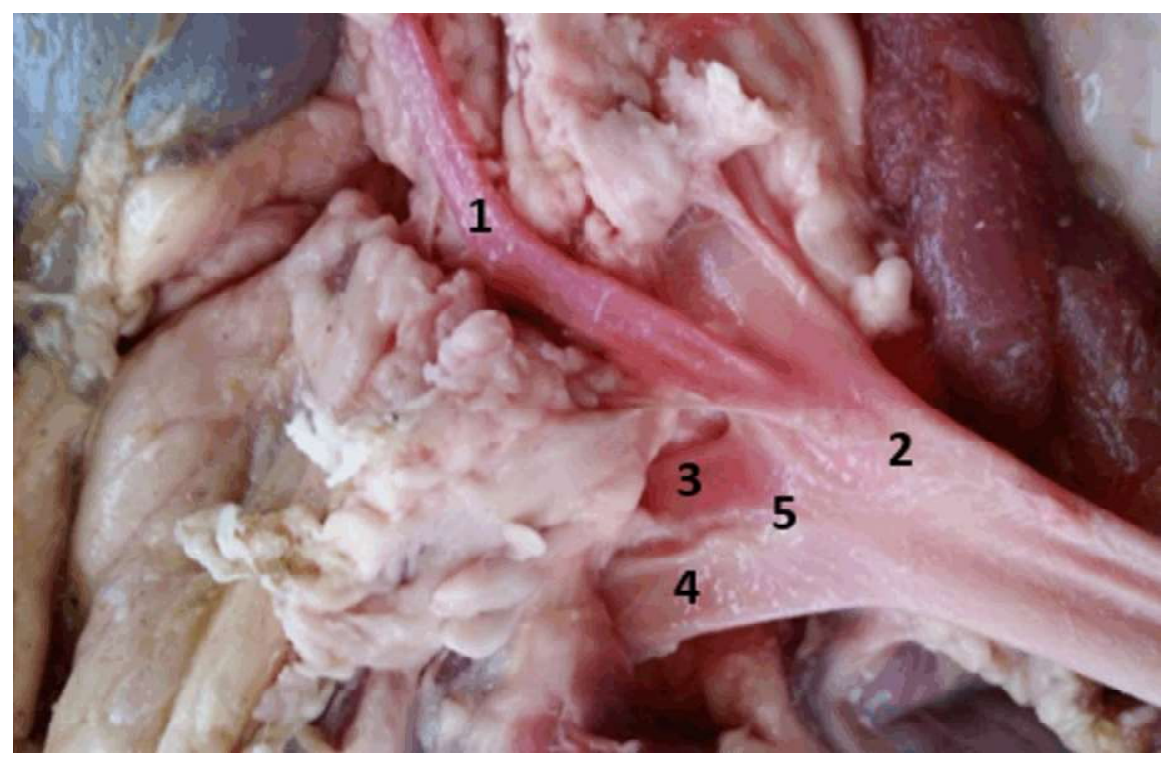

Figura 3. Origen de las arterias mesentérica craneal y tronco celiaco en la alpaca. (1) Arteria renal: (2) arteria aorta abdominal: (3) mesentérica craneal; (4) tronco celiaco; (5) origen común arterial

En estudios realizados a personas que viven sobre los $2000 \mathrm{msnm}$ y con baja presión de oxígeno se observó una adaptación metabólica que les permite realizar actividad física con normalidad en comparación a individuos de la costa (Gonzales, 2001). También se observó una hipertensión asintomática con aumento del grosor de la túnica media en arterias pulmonares, con la capa muscular extendiéndose hasta las arteriolas, aumentando la resistencia vascular pulmonar como una respuesta adaptativa (Peñaloza, 2012). Los animales que viven a estas altitudes como la alpaca, no estarían exentos de adaptaciones en sus capas arteriales de órganos importantes como el páncreas para compensar las duras condiciones ambientales.

\section{Conclusiones}

- Se confirmó que el páncreas de la alpaca está irrigado principalmente por la arteria pancreática duodenal craneal y pancreática duodenal caudal, así como

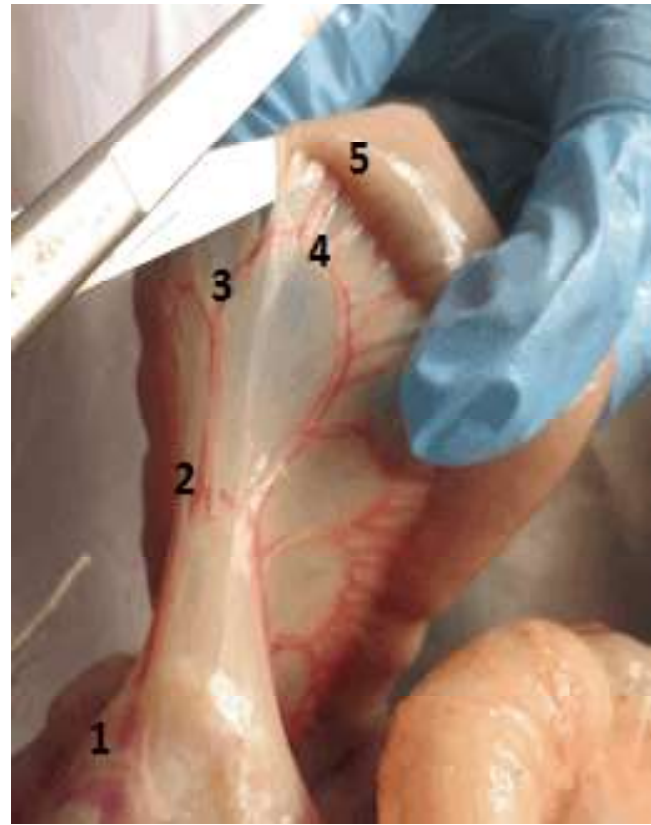

Figura 4. Arterias que irrigan al yeyuno de la alpaca. Se observa la distribución de las ramas arteriales yeyunales. (1) Arteria yeyunal; (2) anastomosis entre las arterias yeyunales; (3) formación de arcos arteriales yeyunales; (4) ramitas rectas arteriales; (5) yeyuno 


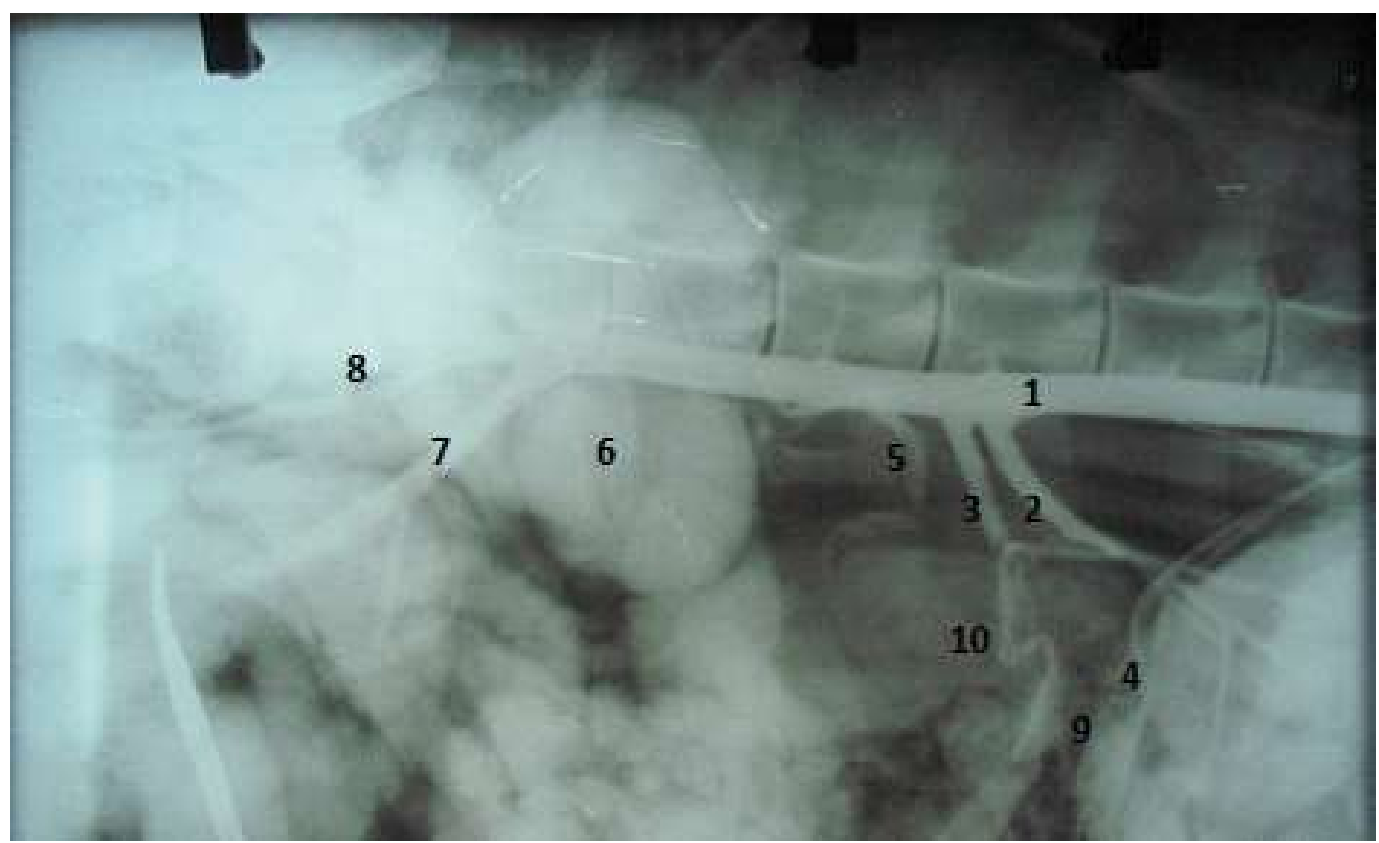

Figura 5. Angiografía de las arterias que emergen de la arteria aorta abdominal de una alpaca adulta (1) arteria aorta abdominal; (2) arteria tronco celiaco; (3) arteria mesentérica craneal; (4) arteria pancreático duodenal craneal; (5) arteria renal; (6) arteria mesentérica caudal; (7) arteria iliaca externa; (8) arteria iliaca interna; (9) arteria pancreático duodenal caudal; (10) arteria yeyunal

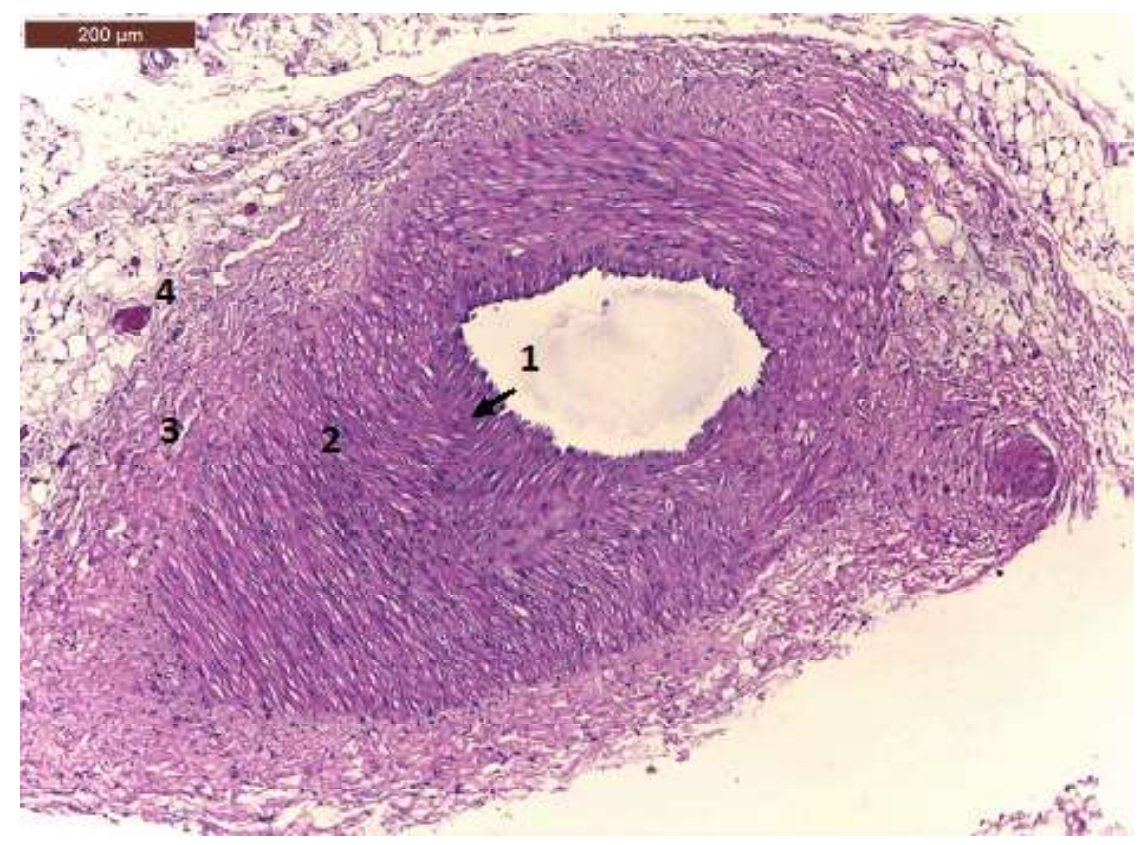

Figura 6. Corte histológico de la arteria pancreática duodenal craneal de la alpaca adulta. (1) túnica íntima; (2) túnica media; (3) túnica adventicia; (4) vasa vasorum (H-E 100X) 


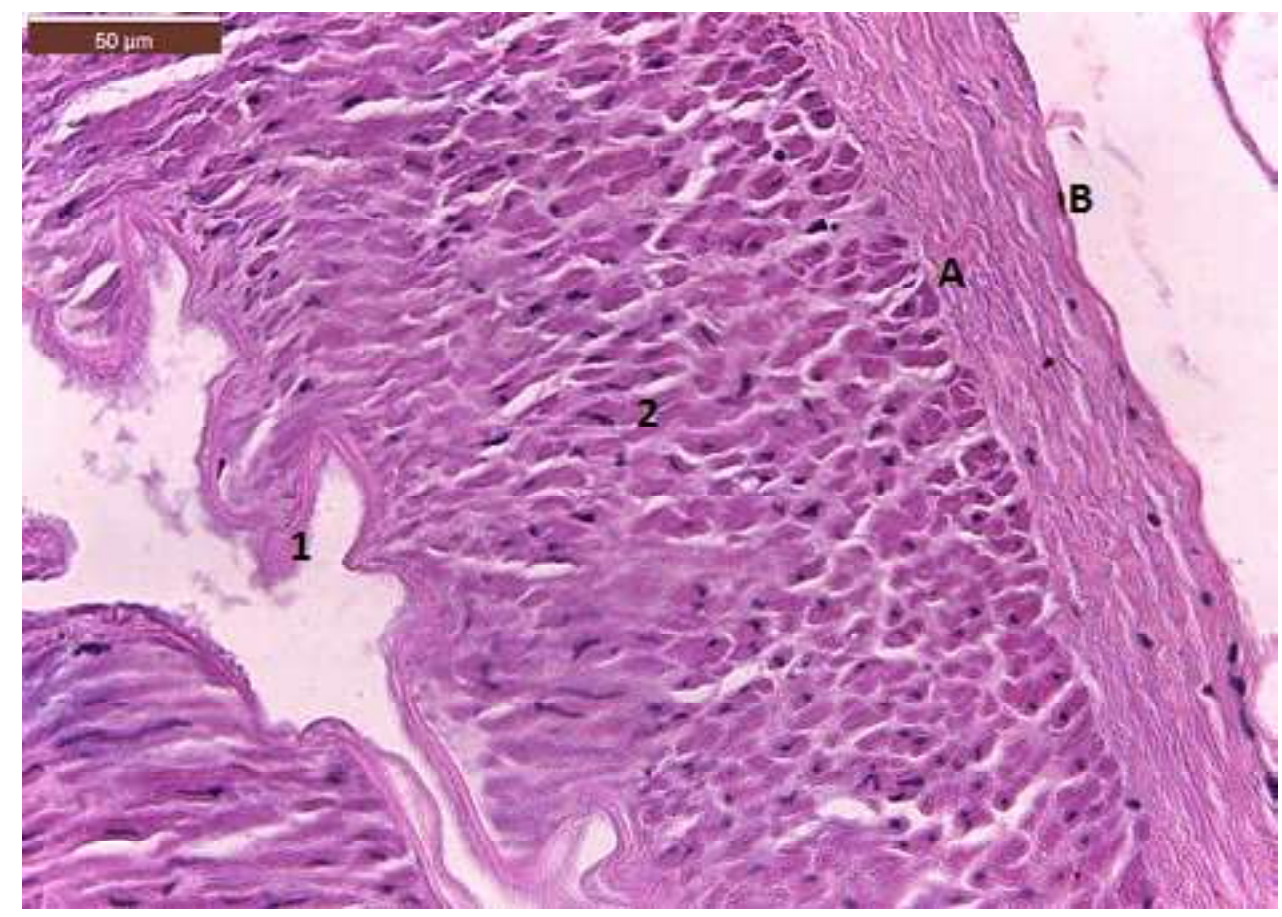

Figura 7. Corte histológico longitudinal de la arteria pancreática duodenal caudal de alpaca. Corte longitudinal. (1) capa endotelial; (2) capa muscular; (A) capa adventicia densa; (B) capa adventicia laxa (HE 400X)

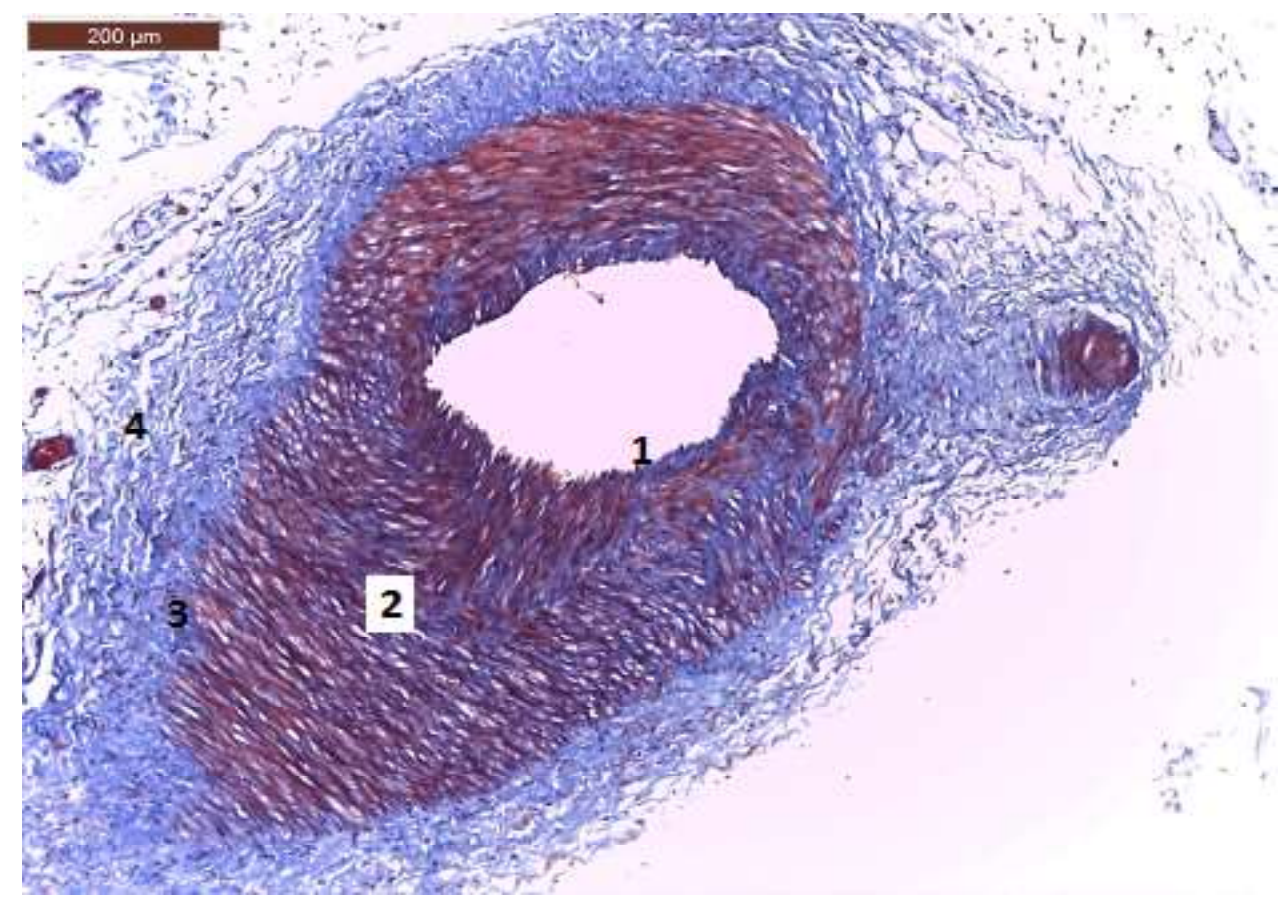

Figura 8. Corte histológico transversal de la arteria pancreática duodenal craneal de la alpaca adulta (1) túnica íntima (2) túnica media (3) túnica adventicia (4) vasa vasorum (tricrómico de Masson 100X). 


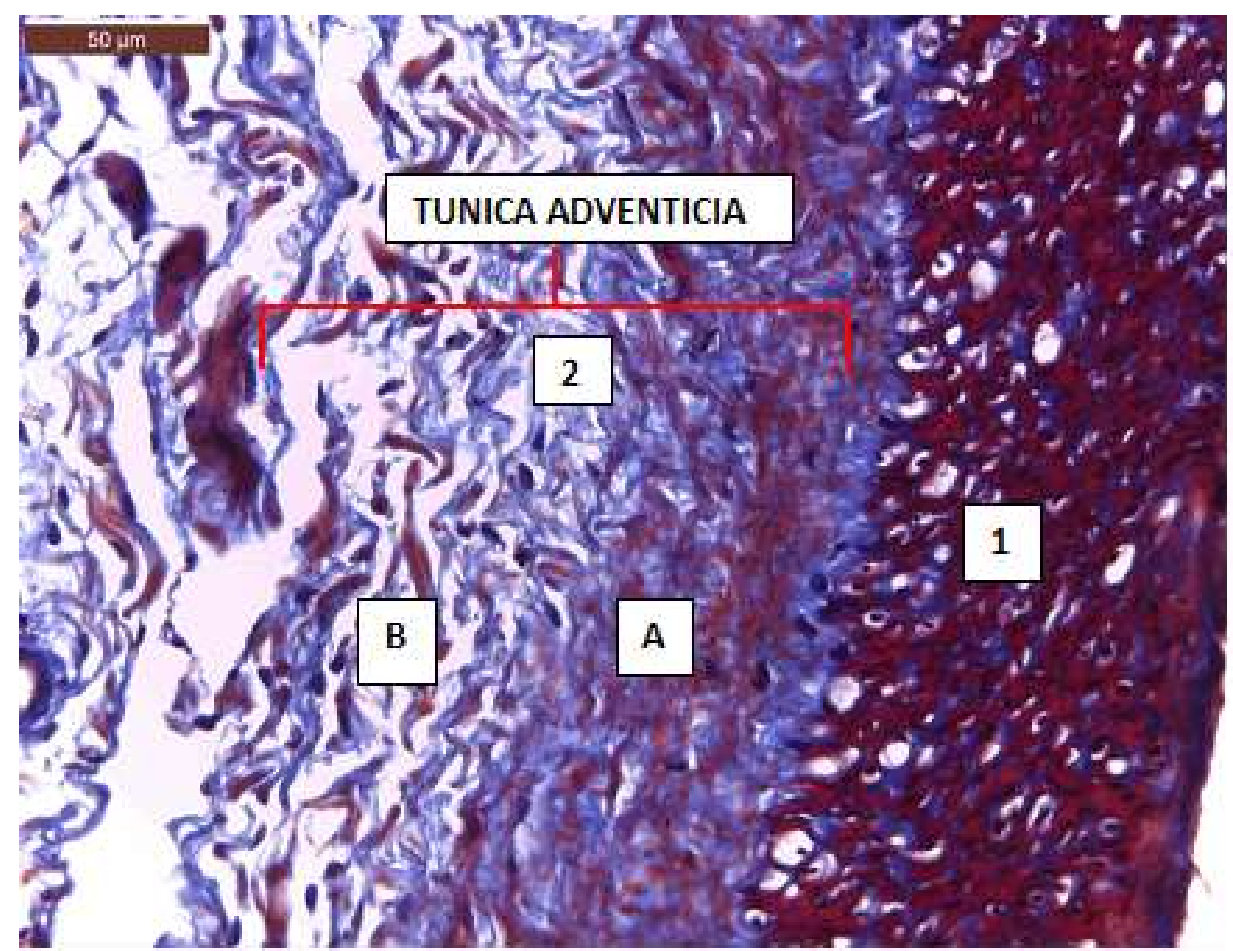

Figura 9. Corte histológico transversal de la arteria pancreática duodenal caudal de alpaca. (1) túnica media: (2) túnica adventicia; (A) capa adventicia compacta; (B) capa adventicia laxa (tricrómico de Masson) $400 \mathrm{X}$

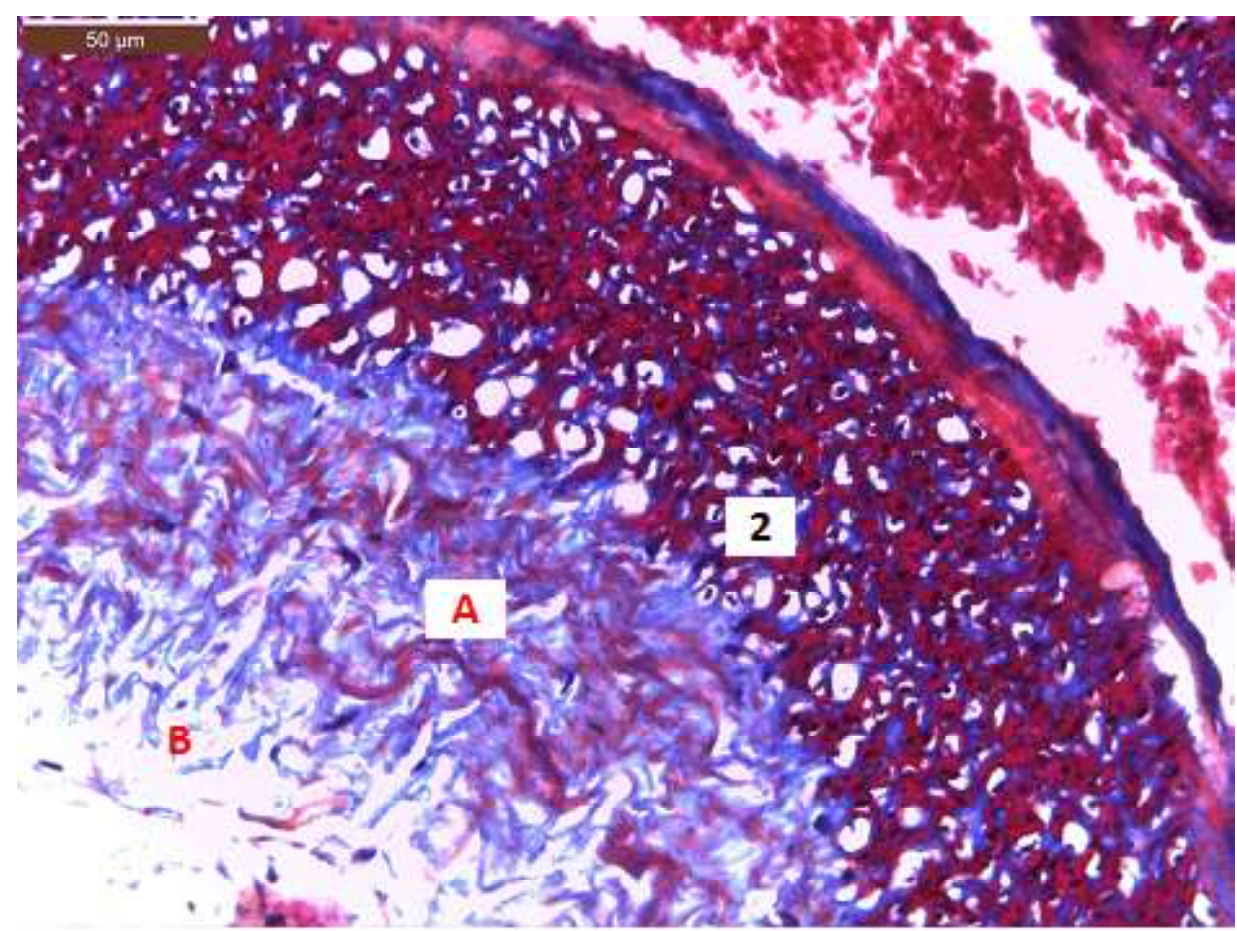

Figura 10. Corte histológico transversal de la arteria pancreática duodenal caudal de alpaca. Corte longitudinal (1) capa endotelial; (2) capa muscular; (A) capa adventicia densa; (B) capa adventicia laxa (tricrómico de Masson) 400X 
por ramas pequeñas de las arterias hepática, esplénica y yeyunal.

- La arteria pancreática duodenal craneal irriga el lóbulo izquierdo, mientras que la arteria pancreática duodenal caudal irriga el lóbulo derecho y parte del cuerpo del páncreas.

- Histológicamente, las arterias pancreática duodenal craneal, caudal, celíaca y mesentérica craneal presentan dos capas, compacta y esponjosa, a nivel de la túnica adventicia. Además, la arteria pancreática duodenal caudal presenta la capa compacta más gruesa a nivel de la túnica adventicia en comparación con la arteria pancreática duodenal craneal.

\section{Agradecimientos}

Los autores agradecen al FONDECYT CONCYTEC por su financiamiento al presente estudio mediante el convenio 097-2014.

\section{Literatura Citada}

1. Cabrera EI. 2008. La adventicia: estado actual del conocimiento. Rev Argentina Cardiol 76: 58-63.

2. Cebra C, McKane S, Tornquist S. 2001. Effects of exogenous insulin on glucose tolerance in alpacas. Am J Vet Res 62: 1544-1547. doi: 10.2460/ ajvr.2001.62.1544

3. Cediel F, Cárdenas M, García A, Chuaire L, Payan C, Villegas V, Sánchez C. 2009. Manual de histología, tejidos fundamentales. Bogotá: Univ. del Rosario. $256 \mathrm{p}$.

4. Cooney K, Chappell J, Callan R, Connally B. 2012. Veterinary euthanasia techniques. A practical guide. Iowa, USA: Wiley-Blackwell. 198 p.

5. Dyce K, Sack W, Wensing C, Larios P, Aja S, et al. 2007. Anatomía veterinaria. $3^{\text {a }}$ ed. México DF: El Manual Moderno. 920 p.
6. Evans H, De Lahunta A. 2002. Disección del perro. $5^{\mathrm{a}}$ ed. México DF: McGraw-Hill. 384 p.

7. Gázquez A, Rodríguez A. 2004. Tratado de histología veterinaria. Barcelona: Masson. 462 p.

8. Gonzales GF. 2001. Metabolismo en las grandes alturas. Acta Andina 9: 31-42.

9. [ICVGAN] International Committee on Veterinary Gross Anatomical Nomen-clature. 2012. Nómina anatómica veterinaria. $5^{\text {a }}$ ed. rev. Missouri: Editorial Committee. $223 \mathrm{p}$.

10. Kierszenbaum A, Tres L. 2013. Histología y biología celular. $3^{\mathrm{a}}$ ed. Barcelona: Saunders. $718 \mathrm{p}$.

11. Laos A. 1973. Irrigación arterial de los compartimentos del estómago de la alpaca (Lama pacos). Tesis de Médico Veterinario. Lima, Perú: Univ. Nacional Mayor de San Marcos. 45 p.

12. Lis A, Barra F, Beltramino F, Peralta J, Rejf P. 2003. Páncreas: diferencias microestructurales y de indicadores funcionales. Sitio Argentino de Producción Animal. [Internet]. Disponible en: http:// www.produccionanimal.com.ar/informacion_tecnica/destete/58-pancreas.pdf

13. Mendoza G, Montoya L. 2002. Anatomía macroscópica de los músculos del cuello de la alpaca. Rev Inv Vet Peru 13: 6-16. doi: 10.15381/rivep.v13i1.1701

14. Navarrete M, Guezzi M, Chávez, A, Sato A, Santos F, Castañeda H, Buitrón L. 2015. Descripción macroscópica de las arterias y venas del páncreas de la alpaca (Vicugna pacos). En: VII Congreso Mundial en Camélidos Sudamericanos. Puno, Perú: Univ. Nacional del Altiplano.

15. Olivos E. 1974. Irrigación arterial del intestino delgado y grueso de la alpaca (Lama pacos). Tesis de Médico Veterinario. Lima, Perú: Univ. Nacional Mayor de San Marcos. 23 p.

16. Panesi G 2016. Estudio morfológico del páncreas en alpacas (Vicugna pacos) jóvenes y adultas. Tesis de Médico Ve- 
terinario. Lima, Perú: Univ. Nacional Mayor de San Marcos. 28 p.

17. Peñaloza D. 2012. Efectos de la exposición a grandes alturas en la circulación pulmonar. Rev Española Cardiol 65: 1075-1078. doi: 10.1016/j.recesp.2012.06 .027

18. Santos F, Ghezzi M, Chávez A, Castañeda H, Sato A, Navarrete $Z$. 2015. Anatomía descriptiva de las arterias y venas de los órganos mesentéricos de la alpaca (Vicugna pacos). En: VII Congreso Mundial en Camélidos Sudamericanos. Puno, Perú: Univ. Nacional del Altiplano.
19. Sato A. 1970. The brain of the alpaca (Lama pacos). An anatomical study. MSc Thesis. Davis, USA: University of California. $128 \mathrm{p}$.

20. Sato A, Kian OT. 1976. Aspectos morfológicos del cerebelo de la alpaca (Lama pacos). Anat Histol Embryol 5: 105-112. doi: 10.1111/j.1439-0264.1976.tb00777.x

21. Sato A, Kitsutani G, Guzmán J. 1983. Inervación periférica de los segmentos del hombro y brazo de la alpaca (Lama pacos). UNMSM 2: 44. 